\title{
CORRECTIONS
}

\section{Benign male genital dermatoses}

Two oversights occurred in this clinical update (BMJ 2016;354:i4337 doi:10.1136/bmj.i4337). In table 4, the clinical features of warts should have been described as: "Commonly presented as soft cauliflower-like growths of varying size; less commonly, flat [not flay], plaque-like, or pigmented. In the section entitled "Biopsy," it should have said "a biopsy result positive for lichen sclerosus or penile intraepithelial neoplasia does not exclude [not excludes] invasive cancer at another site." 